\title{
La ciudad deportiva: un modelo integrador y movilizador The sports city: an integrating and mobilizing model
}

\author{
Carlos Diago, Diana Piedrahita, Jhon Santos, Juan Luis Zapata \\ Alcaldía Distrital de Santiago de Cali, Secretaría del Deporte y la Recreación (Colombia)
}

\begin{abstract}
Resumen. Las denominadas ciudades deportivas se configuran como una especialización inteligente que va más allá de la capacidad de la localidad para realizar grandes eventos y megaeventos deportivos. Ser una ciudad deportiva implica la unión de diferentes stakeholders del deporte a través de diversas políticas públicas encaminadas a potencializar la transversalidad del deporte como herramienta que promueve el bienestar de los ciudadanos. Para los efectos de este propósito, se ha desarrollado una investigación cualitativa basada en la teoría fundamentada, proceso de carácter inductivo donde son los datos y su profundización los que orientan el desarrollo de la perspectiva teórica. Los resultados permitieron conceptualizar la ciudad deportiva y la aproximación hacia un modelo integrador y movilizador el cual está compuesto por: pilares, sistema, actores, impacto y como eje central el deporsistema donde interactúan y se interrelacionan cinco componentes bióticos/abióticos, materiales o conceptuales que cumplen un propósito y como en la Gestalt «el todo es más que la suma de las partes». A través de este modelo de ciudad, se pretende generar un instrumento proyectivo, facilitador del aseguramiento de estrategias que guiarán a la gobernanza del deporte en el diseño de un modelo propio adaptado al entorno, oportunidades, retos, fortalezas, necesidades y prioridades de la población. Ratificando la relevancia del deporte en la planificación a largo plazo de la ciudad contemporánea.
\end{abstract}

Palabras Clave: ciudad, deporte, políticas, estrategias, modelo, deporsistema.

\begin{abstract}
The purpose of this article is to highlight how the so-called sports cities have managed to become an intelligent specialization that goes beyond the disposition of the city to carry out large sports events and mega-events. Being a sports city implies the union of different sports stakeholders through various strategies and public policies aimed at strengthening the mainstreaming of sport as a tool that promotes the well-being of citizens. For the purposes of this purpose, a qualitative research based on grounded theory has been developed, an inductive process where the data and its deepening are the ones that guide the development of the theoretical perspective. The results allowed the definition of a sports city and the approach towards an integrating model which is composed of: pillars, system, actors, impact and as the central axis the deporsystem where five biotic / abiotic, material or conceptual components interact and interrelate. they serve a purpose and as in Gestalt «the whole is more than the sum of the parts». Through this city model, it is intended to generate a projective instrument, facilitating the assurance of strategies that will guide sport stakeholders, in the design of their own model adapted to their context, needs and desires of the population, in addition to the forward-looking governance planning. Ratifying the importance of sport in the future planning of the contemporary city.
\end{abstract}

Keywords. city, sport, policies, strategies, model, sports system.

\section{Introducción}

En algunas ciudades contemporáneas, la especialización inteligente es oportunidad para aumentar competitividad y productividad, mediante el abordamiento de un planteamiento estratégico dando respuesta a grandes desafíos de la sociedad actual (Landabasco, 2015). De ahí, el top cinco del «Indicador de ciudades globales»: Nueva York, Londres, Paris, Tokio y Beijiing. Además del posicionamiento de «ciudad perfecta» dada a otras ciudades como: New York para los negocios y el capital humano, Paris para el intercambio de información, Londres para las experien-

Fecha recepción: 03-04-21. Fecha de aceptación: 27-09-21

diana.piedrahita@cali.gov.co

Diana Piedrahita cias culturales, Los Ángeles para la realización de eventos deportivos, Bruselas para la participación política (Kearney, 2020).

En ese mismo sentido la UNESCO proyecta el mejoramiento de las ciudades, mediante estrategias cimentadas en el aporte de algunos factores determinados al logro de los Objetivos de Desarrollo Sostenible (ODS) como lo pueden ser las ciudades creativas, ciudades del aprendizaje, alianza de megaciudades por el agua y el clima (UNESCO, 2020)

Es así como existen diversas ciudades que presentan enfoques relacionados con la importancia que el sector deporte tiene como capital social y económico debido a que este además de inspirar, conectar, comprometer, involucrar, evocar emociones, como sector económico, a pesar de las importantes discrepancias que surgen al intentar medirlo, se le reconoce su rápido crecimiento. 
Respecto a la medición del valor del sector, la consultora ATKearney reportó que para el 2014, dicha industria generó aproximadamente USD700 billones anuales, lo que representó un 1\% del PIB mundial.

Precisamente este contenido refiere a ciudades que utilizan como estrategia la especialización inteligente basada en el deporte, en una mirada hacia la imagen creciente de las urbes modernas que han visto en este una herramienta para realizar importantes transformaciones de ciudad de índole socio-económico, las cuales son fundamentadas en políticas públicas que permitieron cambios en los cuales la gobernanza pública es el componente planificador y articulador de los modelos de administración deportiva de la denominadas sports cities o ciudades deportivas.

Retomando un poco de historia sobre las raíces de las ciudades deportivas, se pueden ubicar tres momentos importantes, el primero se origina en Grecia clásica, organizadora de los Juegos Panhelénicos.

Dichos Juegos eran competiciones atléticas de carácter sagrado realizadas en las ciudades santuarios de Delfos (Juegos Píticos), Corinto (Juegos Ístmicos), Argos (Juegos Nemeos) y Olimpia donde se celebraban los Juegos Olímpicos (JJ OO) de la antigüedad que datan del 776 aC. El segundo momento refiere a tiempos modernos, donde la orientación como ciudades deportivas se le atribuye a Roma con la realización de los JJ OO de 1960 (Muñoz, 2006; Whitson, 2004) cuando por primera vez un megaevento se posiciona como instrumento de desarrollo urbano, lo que Muñoz (1997) denomina urbanismo olímpico debido a que las competiciones se realizaron en lugares icónicos de la ciudad, atractivos referentes de su importante pasado arquitectónico. El tercer momento se ubica en los JJ OO de Barcelona 1992 reconocidos por su rol preponderante en la modernización de los JJ OO.

En la actualidad, cada vez más la gobernanza pública reconoce el deporte en términos de creación de nuevas fuentes para la economía (Gratton, et al., 2005), la contribución que hace el deporte y las instalaciones deportivas para la habitabilidad de la ciudad, así mismo, se evidencia una creciente conciencia social sobre el potencial del deporte para el desarrollo (Coalter 2005, 2007a, 2007b) sobre todo cuando se trata de programas locales (Coalter, 2010). Todo esto confluye en el auge de las ciudades deportivas consideradas una de las últimas manifestaciones del deporte mundial (Pye, et al., 2015).

Para los efectos de este propósito se ha desarrollado una investigación cualitativa basada en el método de la
Teoría Fundamentada (TF) que para Strauss \& Corbin (2002,p22) trata de teorizar acerca de un fenómeno cuando el investigador carece de una preconcepción del tema, por tanto «la recolección de datos, el análisis y la teoría que surgirá de ellos guardan estrecha relación entre sí».

Debido a esto el muestreo teórico y el proceso inductivo, igual que la codificación y la profundización en la analítica de datos, es lo que finalmente permitió a los investigadores determinar los casos objeto de análisis acorde a su nivel relacional con los conceptos y teorías ya desarrollados. De esta manera a partir de teorización que surge de la información recolectada se presenta un modelo de integrador de lo que puede ser una ciudad deportiva.

\section{Antecedentes}

En años recientes, algunas ciudades han encadenado una serie de facilidades concentradas en determinadas áreas, donde se diseñan, crean o revitalizan zonas deportivas temáticas en un lugar específico de la urbe, o han calificado toda una ciudad como «Ciudad Deportiva» a través de estrategias relacionadas con marca de ciudad y la especialización inteligente para potenciar el desarrollo en torno al valor del deporte, siendo a su vez coherentes con el posicionamiento a través del deporte que la ciudad desea ofrecer.

Otras ciudades tienen estrategias relacionadas con hacer a la ciudad más atractiva para hospedar equipos profesionales o eventos deportivos, racionalizar costos de construcción de infraestructura deportiva, incrementar el beneficio económico de un gran evento deportivo, atraer turistas, fomentar la inversión interna, regenerar la imagen e incrementar el empleo en ciudades que perdieron alguna de sus vocaciones.

Es por ello que diversas organizaciones como las siguientes, han categorizado las ciudades en referencia bajo nominaciones específicas, etiquetándolas como «ciudades deportivas»:

Dichos rankings tienen en común la importancia dada a la realización de eventos deportivos especialmente grandes eventos y megaeventos, sin embargo, no ofrecen ninguna definición de lo que es una Ciudad Deportiva, de otro lado, utilizan mecanismos de medición diferentes por lo que se dificulta compararlos entre sí, sin embargo, aportan diferentes aspectos que deben considerar las ciudades deportivas como lo es la realización de grandes eventos deportivos, la infraestructura, la presencia de organizaciones deportivas, el marketing y 


\begin{tabular}{|c|c|c|c|}
\hline Organización & Etiqueta & Top tres Latinoamérica (LATAM) & Factores \\
\hline $\begin{array}{l}\text { BCW (Burson Cohn \& } \\
\text { Wolfe) }\end{array}$ & $\begin{array}{l}\text {-Ranking de ciudades deportivas (top 50). } \\
2021 \text { New York }\end{array}$ & $\begin{array}{l}\text { Rio de Janeiro (27). } \\
\text { Buenos Aires (44) }\end{array}$ & $\begin{array}{l}\text { Opiniones de las federaciones internacionales, expertos en deporte, medios deportivos } \\
\text { y la asociación entre deporte y ciudad en entornos digitales. }\end{array}$ \\
\hline Ranking of sports cities & $\begin{array}{l}2020 \text { New York } \\
2019 \text { Londres } \\
2018 \text { Londres }\end{array}$ & & Selecciona a la ciudad que tenga la asociación más fuerte entre deporte y ciudad. \\
\hline ACES Europe & $\begin{array}{l}\text {-World Capitals of Sport } \\
\text { 2020-2021: Guadalajara } \\
\text { 2019 Abu Dhabi } \\
\text {-European Capitals of Sport } \\
\text { 2020 Málaga } \\
\text { 2021 Lisboa }\end{array}$ & Cali: Capital Americana del Deporte 2019. & $\begin{array}{l}\text { Las ciudades se postulan. La información analizada es la siguiente: } \\
\text {-Información general de la ciudad. } \\
\text {-Instalaciones e infraestructura deportiva. } \\
\text {-Acceso a instalaciones y actividades deportivas. } \\
\text {-Deporte asociado y voluntariado. } \\
\text {-Eventos realizados por la administración local. } \\
\text { - Promoción del deporte y salud y deporte en todas las clases sociales. } \\
\text {-Promoción del deporte inclusivo. } \\
\text {-Indice de deportividad de la población. } \\
\text {-Movilidad } \\
\text {-Turismo deportivo. } \\
\text {-Comunicación } \\
\text {-Relaciones entre la organización local y organizaciones del deporte. } \\
\text {-Actividades como capital deportiva. } \\
\text {-Calendario anual de actividades deportivas. }\end{array}$ \\
\hline $\begin{array}{l}\text { Sportcal/-Global Sport } \\
\text { Impact (GSI) } \\
\text {-Global Sports Impact Cities }\end{array}$ & $\begin{array}{l}\text {-Ranking Global Sport Impact Cities 2019: } \\
\text { Tokyo }\end{array}$ & $\begin{array}{l}\text {-Rio de Janeiro (4) } \\
\text {-Lima (17) } \\
\text {-Buenos Aires (56) } \\
\text {-Cali } 88\end{array}$ & $\begin{array}{l}\text { Para el } 2019 \text { el GSI Cities Index analizó en profundidad } 730 \text { grandes juegos deportivos y } \\
\text { campeonatos mundiales durante un período de } 14 \text { años para el ranking } 2019 \text { ( } 2014- \\
\text { 2024), se analizaron } 83 \text { deportes, } 730 \text { eventos, } 156 \text { categorías, } 641 \text { ciudades de } 90 \\
\text { naciones. }\end{array}$ \\
\hline $\begin{array}{l}\text { Sport Business } \\
\text { Varias categorías } 2018\end{array}$ & $\begin{array}{l}\text {-Ciudad Deportiva Internacional por } \\
\text { Excelencia 2018: Londres } \\
\text {-Mejor gran ciudad: Melbourne } \\
\text {-Extraordinaria gran urbe: Nueva York } \\
\text {-Otras categorías } \\
\text { Mejor transporte: Paris. } \\
\text {-Mejor alojamiento: Dubai. } \\
\text {-Mejor estrategia de eventos: Melbourne } \\
\text {-Mejor legado: Londrés } \\
\text {-Mejor marketing y branding: London } \\
\text { Mejor nuevo en la categoría: Abu Dhabi }\end{array}$ & $\begin{array}{l}\text { SportBusiness International Ultimate } \\
\text { Sports City 2018: } \\
\text {-México (24) } \\
\text {-Río de Janeiro (25). }\end{array}$ & $\begin{array}{l}\text {-Número de federaciones y cuerpos administrativos radicados en la ciudad y su } \\
\text { importancia. } \\
\text {-Sedes e instalaciones actuales, y capacidades para grandes eventos deportivos } \\
\text {-Transporte e infraestructura } \\
\text {-Alojamiento } \\
\text {-Apoyo gubernamental y estrategia para la realización de importantes eventos. } \\
\text {-Planificación e impacto del legado. } \\
\text {-Interés público y asistencia a eventos } \\
\text {-Marketing y branding }\end{array}$ \\
\hline
\end{tabular}

la capacidad de atender los grandes eventos.

Para Pye et al. (2015) lo que las ciudades deportivas tienen en común no es solo una fuerte afiliación con el deporte, sino también la intención de aprovechar esta conexión para alguna forma de ventaja económica, social o reputacional, por esto recomiendan que la planificación de una Ciudad Deportiva las necesidades sociales, económicas y ambientales de la comunidad mientras maximiza el potencial económico de la industria mundial del deporte la cual continua en permanente expansión.

Dicho crecimiento es posible debido a la diversidad de apuestas que se pueden realizar en torno al deporte como lo expresan Nauright \& Zipp, 2018 (p.1114) «El deporte es frecuentemente elogiado por unir a las personas, herramienta útil para el desarrollo y paz, y un mecanismo para abordar las preocupaciones de salud y fitness en sociedades que enfrentan aumento de los costos de la atención médica».

Es por esto que el objetivo de ser Ciudad Deportiva puede diferir entre un caso y otro; en el Reino Unido y EE U, zonas de la ciudad se utilizan para regenerar terrenos con poco uso y reorientar economías urbanas hacia los sectores del ocio y el turismo deportivo, tal es el caso de Cardiff, Manchester, Glascow, Indianápolis. Baltimore, Memphis. En ciudades de Oriente Medio como es el caso de Doha y Dubái, grandes proyectos de desarrollo urbano, incorporan zonas deportivas que tienen como objetivo el desarrollo de atletas de altos lo- gros, ser futura sede de grandes eventos y megaeventos deportivos, atraer turistas y/o nuevos residentes con alto poder adquisitivo. En el caso de los Ángeles y Melbourne, las alianzas público privadas para la realización de grandes eventos deportivos son el eje fundamental de la «Ciudad Deportiva».

Otro rubro de la economía del deporte que aporta a la Ciudad Deportiva son las tiendas de equipamiento deportivo, las cuales han representado un gran aporte a la economía del deporte durante la pandemia por la enfermedad Covid-19.

Es el caso de EE UU donde el comercio de equipamiento deportivo comenzó el 2020 con alzas entre el $6 \%$ y $7 \%$, con la pandemia las ventas cayeron un $31 \%$ en abril, pero en mayo inició la recuperación y continuaron creciendo hasta lograr un mayor crecimiento con respecto al 2019, logrando en los últimos meses

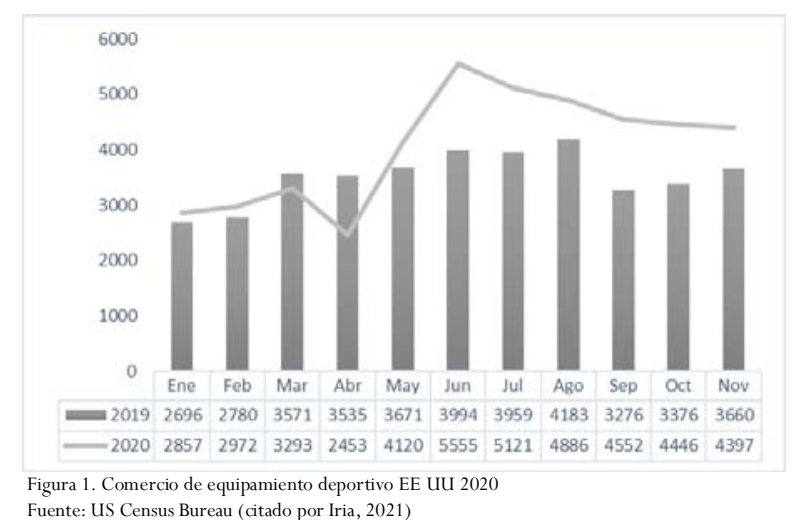


del año alzas entre el 20\% y el 30\%, mientras el conjunto del comercio apenas superaba el $6 \%$ como es el caso de la moda y la hostelería quienes cerraron el año con desplomes del $26.4 \%$ y del $19.5 \%$, respectivamente (Iria, 2021).

Otro producto destacado en estas urbes son las tiendas temáticas las cuales funcionan como atractores del turismo más que como tiendas minoristas, son relevantes debido a que pueden gestionarse como una mezcla de actividades en las que se puede participar y realizar exhibiciones físicas, mientras el plan de medios puede ser diseñado para recrear un ambiente deportivo temático atractivo para turistas (Smith, 2010). Este canal requiere segmentar la tienda por áreas temáticas, talento humano capacitado en cada una de las áreas, living lab como lo puede ser el análisis de la pisada o del pedaleo, probadores de nuevas tecnologías, activaciones para eventos deportivos. Recursos cuyo propósito es solucionar necesidades muy concretas en torno a retos de afluencia (atraer más personas al interior de la tienda) y de influencia (convencer más personas para comprar productos).

Adicional a esto, la Ciudad Deportiva debe abordar temas relacionados con la buena gobernanza y la responsabilidad social, para Naurigth \& Zipp (2018), en la actualidad, el sistema global del deporte no es sostenible, su viabilidad depende de la realización de cambios y transformaciones como lo ha sido la lucha por erradicar la corrupción de muchas organizaciones que ha hecho posible procesos de licitación cada vez más transparentes, sin embargo quedan retos como el dopaje, arreglo de partidos, abuso infantil y el abuso sexual. Favorece que la mayoría de la gente, a pesar de la evidencia, todavía cree en el deporte como una actividad neutral que atrae multitudes (Moeller \& Nauright, 2003).

En consecuencia, existe reconocimiento acerca de ciudades como Birmingham, Manchester y Sheffield que han hecho del deporte una herramienta para reimaginar la ciudad y como es el caso Barcelona perennizar el espíritu olímpico (Smith, 2005a,b).

Para algunos es «difícil comprender lo qué es una ciudad del deporte» (Smith, 2005a,p.229) y a pesar de la amplia mención que se hace, es un «concepto bastante abstracto» (Smith, 2010,386).

\section{Modelo integrador y movilizador para una Ciudad Deportiva}

Si bien, es claro el propósito de establecer el aporte del deporte para el desarrollo sostenible de la ciudad contemporánea por su capacidad para influir en aspectos como las dinámicas de raza, género, religión, etnia, sexualidad, discapacidad, economía, desarrollo sostenible, estilo de vida saludable, transmisión de valores, confianza en las instituciones y el poder que tiene para trascender por encima de fronteras regionales y nacionales debido a sus competencias para integrar (Biernat et al.,2019; Brown et al., 2014; Deloitte, 2020; Donnelly, 2010; Janssens \& Verweel, 2014; Naurigt \& Zipp, 2019).

Es necesario para este propósito, definir conceptualmente el alcance de una Ciudad Deportiva, entendiendo que, Ciudades Deportivas son aquellas urbes en las cuales la gobernanza pública ha valorado el deporte como fenómeno social multifacético, imprescindible para el logro de su desarrollo sostenible a nivel socio-cultural, económico y ambiental mediante el fortalecimiento y la animación del derecho al deporte y la recreación entre toda la población y a lo largo de toda la vida.

Por esto, con miras a establecer un modelo sostenible de Ciudad Deportiva, se tendrá como referente, temas tomados en cuenta por diferentes organizaciones para establecer rankings para dichas ciudades, aspectos desarrollados por otras ciudades deportivas y consideraciones propias entre las cuales se encuentra integrar en un solo concepto todos los componentes que a nuestro juicio son inherentes a una Ciudad Deportiva y al que hemos denominado deporsistema.

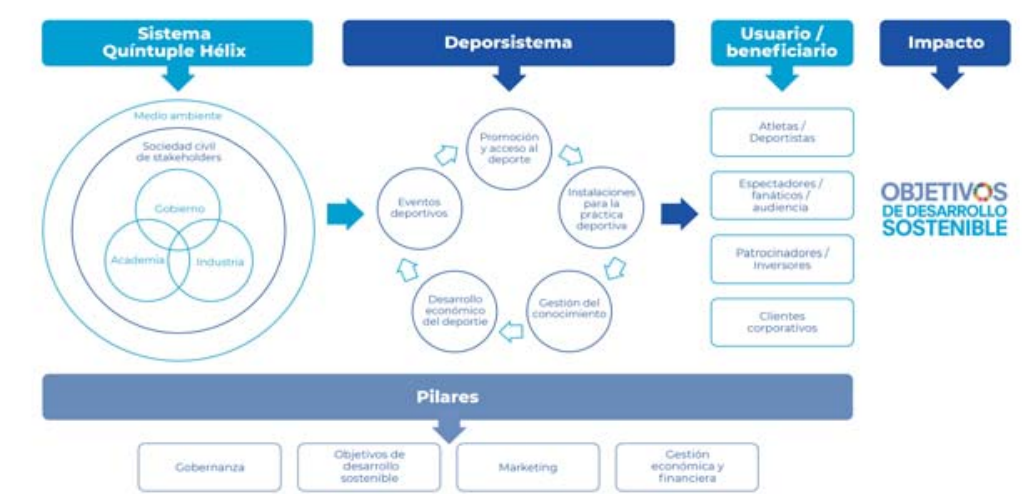

Figura 2. Modelo integrador y movilizador de una Ciudad Deportiva

El esquema refiere cada uno de los elementos constitutivos del modelo de Ciudad Deportiva propuesto, cuyos cimientos son cuatro pilares relacionados con I a planificación y la gestión. A modo de processo, las entradas estarían ofertadas por el sistema cuyos actores refieren al modelo de la Quíntuple Hélix; el proceso propiamente dicho sería el Deporsistema conformado por cinco factores que son indispensables para el desarrollo integral de la Ciudad Deportiva, quienes, integrados entre todos y unos con otros, tienen la responsabilidad de movilizar todos los componentes del Deporsistema. Las salidas de los componentes del Deporsistema tienen como objetivo lograr la satisfacción de los diferentes usuarios/beneficiarios, finalmente el impacto es la medición de la contribución que el deporte realiza al desarrollo sostenible y sustentable de la ciudad y por ende al bienestar de la ciudadanía.

Fuente: Elaboración propia. 
Cuanto se reconoce el deporte y el tiempo de ocio como políticas sociales prioritarias (dos Anjos, 2021) se puede decir que la representación cualitativa y cuantitativa del gobierno es la buena gobernanza, el proyecto AGGIS (Acción para la buena gobernanza en organizaciones deportivas internacionales), es una lista de verificación de los elementos de buena gobernanza para las federaciones deportivas internacionales a través de un instrumento de evaluación comparativa basado en el benchmarking: transparencia organizacional, transparencia en los informes, representatividad de las partes interesadas, proceso democrático, mecanismos de control, integridad deportiva, solidaridad (Chappelet \& Mrkonjic,2013). Geeraert (2015) lo representa en cuatro dimensiones transparencia, proceso democrático, separación de poderes y solidaridad las cuales son medidas a través de indicadores.

Gobernanza Debido a esto se deben planificar los beneficios sociales del deporte: incremento en el acceso y oportunidades de práctica deportiva para incrementar la salud fisica y mental del individuo, el desarrollo económico sustentable del sector, espacios públicos que promuevan ciudadanos activos, oportunidades para mejorar la seguridad y contribuir a la reducción del crimen, incentivar la educación y el empleo (Pye et al. 2015). Para Kharytonov, et al. (2020), otro rol de la gobernanza pública es mejorar de manera conjunta a las organizaciones deportivas, los mecanismos de regulación sobre las acciones sociales Para Kharytonov, et al. (2020), otro rol de la gobernanza publica es mejorar de manera conjunta a las organizaciones deportivas, los mecanismos de regulacion sobre las acciones sociales
prioritarias, debido a esto, la política pública que no es otra cosa que la hoja de ruta del gobierno debe ser formulada de manera democrática y participativa (de abajo hacia arriba) y bajo principios de gestión territorial (Castillo-Retamal et al., 2020)

Como pilar, los Objetivos de Desarrollo Sostenible (ODS) representan la necesidad que tiene la gobernanza de articularlos en los planes de desarrollo, en las políticas públicas, planes estratégicos y como indicadores de los planes de acción, realizando trabajo mancomunado con los actores del Deporsistema (sociedad civil, empresas privadas, academia, entidades públicas).

Al respecto, en 2017, durante la octava Reunión de Ministros del Deporte de la Commonwealth (UNESCO \& MINEP VI), se asumió el compromiso de armonizar la arquitectura deportiva y las políticas y estrategias basadas en el deporte con el marco de los ODS y las metas conexas, siendo los siguientes Objetivos de Desarrollo Sostenible especialmente pertinentes:

Sostenible PoDS 3, ODS 4, ODS 5, ODS 8, ODS 10, ODS 11, ODS 16

El desafío actual de la Ciudad Deportiva es incluir en los modelos que desarrollan programas de deporte para el desarrollo sostenible y la paz a "intelectuales orgánicos" (dirigencia o clase política) impregnados de creatividad sociológica que lleven al deporte a trascender de su papel funcionalista a ser un agente activo de cambio social (Naurigth \& Zipp, 2018, p.1114).

\begin{tabular}{|c|c|}
\hline Marketing & $\begin{array}{l}\text { Para el caso, permite desarrollar un conjunto de actividades orientadas a identificar y determinar las necesidades de diferentes públicos reales y potenciales, posicionar y mejorar la imagen de la } \\
\text { Ciudad Deportiva dentro del mercado (stakeholders del deporte), mediante la realización de acciones sociales y transmisión de los principios y valores de la Ciudad Deportiva entre sus } \\
\text { diferentes grupos de interés para mejorar la satisfacción de los ciudadanos. } \\
\text { Marketing de contenidos para la comunicación ciudadana (vídeos, noticias, libros, imágenes, redes sociales). } \\
\text { En consecuencia, dicho posicionamiento tendrá éxito si tanto su ciudadanía como sus empresas se sienten satisfechos con sus comunidades, al mismo tiempo que se atiende correctamente las } \\
\text { expectativas de los visitantes e inversores (Gold \& Ward, 1994) como es el caso de la marca Sport Singapore. }\end{array}$ \\
\hline $\begin{array}{l}\text { Gestión } \\
\text { Económica y } \\
\text { Financiera }\end{array}$ & $\begin{array}{l}\text { El factor económico es un elemento vital para las ciudades deportivas, de su buena gestión depende el éxito el cual está relacionada con principios de buena gobernanza como lo son la rendición } \\
\text { de cuentas y la transparencia. Una buena gestión en este sentido debe incluir aspectos relacionados con la consecución de recursos, la planificación, presupuestos, aplicación y contabilidad, la } \\
\text { evaluación e informes. }\end{array}$ \\
\hline
\end{tabular}

Cabe resaltar que, a través de este modelo de ciudad, se pretende generar un instrumento proyectivo, facilitador del aseguramiento de estrategias que guiarán a los stakeholders del deporte, en el diseño de un modelo propio adaptado a su contexto, necesidades y deseos de la población, además de los de la gobernanza, para lo anterior analizaremos los cinco componentes del que hemos denominado Modelo de Ciudad Deportiva.

\section{Componentes}

Ver tabla 2.

\section{Segundo El sistema - Quíntuple Hélix}

El sistema deportivo, tiene una gran complejidad y de manera frecuente, varia en forma, estructura y propósito en cada país (Gerkea et al., 2018). De ahí que la gestión de vínculos entre organizaciones involucradas en el deporte es cada vez más importante y compleja debido a la heterogeneidad de actividades, metas y resultados (Misener \& Mason, 2010) debido a que para atender las necesidades de los usuarios del deporte, empresas, inversores o visitantes se genera una gran interactividad entre el gobierno local, el sector privado, sector público y la academia.

Con relación al sistema de la Ciudad Deportiva propuesto, este se desarrolla mediante el modelo de la quíntuple hélice y refiere a las interacciones que se dan entre los subsistemas a saber:

El primer subsistema es Estado representado por el gobierno y sus instituciones quienes emiten leyes, le- gislan y ejecutan las acciones del gobierno sobre el territorio. El segundo es la academia que forma el capital humano. El tercero son las empresas e industrias que movilizan y dinamizan la economía. El cuarto es la sociedad civil de stakeholders quienes a través de la cultura y los medios de información establecen el sistema capital social. El quinto conformado por el medio ambiente y los recursos naturales rotulados como capital natural.

\section{Tercero Deporsistema}

Concebido como aquellos componentes inherentes e imprescindibles para la Ciudad Deportiva, cuyo objetivo es contribuir y preservar al desarrollo socio-económico, cultural y ambiental sostenible de todos y cada uno de los stakeholders que conforma la Ciudad Deportiva. Su denominación, se debe a la relación que cada uno de sus componentes tiene con al menos alguno de los demás componentes y su unión representa más que cada una de sus partes, interrelación e interacción de carácter bióticos /abióticos, materiales o conceptuales y como todo sistema, unidos cumplen un propósito, un fin. Es así como se han identificado cinco componentes que se relacionan a continuación.

\section{Promoción y Acceso al Deporte la Recreación y la Actividad} Física

La promoción de la participación universal en el deporte es una importante agenda de políticas públicas en muchos países, por esto una de las principales funciones sociales de la Ciudad Deportiva debe ser garantizar a los individuos el «grado necesario de actividad 
motriz para su plena manifestación en todas las esferas de la vida» (Zhelyazkov, 2006), para esto se hace necesario promover y fomentar en toda la población el deporte para todos, el deporte en edad escolar, el deporte de altos logros, la actividad física, la recreación y el aprovechamiento del tiempo de ocio; mediante la ejecución de programas con una amplia y variada oferta deportiva, direccionada a satisfacer necesidades y deseos de todos los grupos poblacionales en todos los ciclos vitales.

El deporte para todos es una brecha relacionada con la participación que hay que cerrar y superar, debido a que es una condición previa para el «deporte para siempre», con el cual se logran los mayores beneficios sociales. Por ello se deben desarrollar ampliamente los vínculos entre «Deporte para todos»y «Deporte para siempre» para incrementar la contribución del deporte en temas relacionados con prevención del delito, salud, comunidad y economía (Donnelly, 2010; Brown et al., 2014). En resumen, una Ciudad Deportiva debe priorizar beneficios sociales sobre los económicos, consideración a partir de la cual, se han realizado diversos estudios evaluativos para determinar la relación entre la actividad deportiva y el capital social, encontrando aspectos bastante positivos: En adultos mayores de 40 años el deporte es un factor que contribuye al bienestar mediante la construcción de redes sociales, posibilitando que sean socialmente activos y se incremente la percepción acerca de la cantidad de amigos que tienen, por esto se recomienda ampliamente como instrumento para una política de activación social (Skrok et al., 2019).

De igual manera cuando se trata de menores de edad la actividad física y el deporte escolar han demostrado estar fuertemente correlacionados con la formación y desarrollo de capital social debido a los efectos positivos en la salud, el rendimiento escolar y sobre todo en la disminución de problemas sociales debido a que se incrementa el buen comportamiento entre pares. Adicional a esto, cuando la práctica se realiza en un club deportivo, además de reducir el tiempo para actividades pasivas, estar un club reta a tomar la iniciativa para planificar el logro de una meta valiosa a largo plazo, el trabajo en equipo incentiva el valor de la cooperación, lograr la victoria incrementa la autoestima, y la derrota, enseña a resolver situaciones similares (Felfe et al., 2016).

Al respecto, la consultora global Deloitte (2020) recomienda a las organizaciones deportivas asumir el papel fundamental que pueden desempeñar para ayudar a abordar problemáticas sociales relacionadas con raza, perspectiva de género, inequidad e injusticia LGTBI+ como lo espera la sociedad. De esta manera, la justicia social sería un principio de su cultura organizacional y una promesa de marca, esto se logran teniendo presente siempre que las dinámicas sociales son cambiantes y se debe estar preparado para abordarlas.

Otra oportunidad que tienen las organizaciones deportivas es el protagonismo e influencia que están tomando los atletas, quienes ahora prestan su voz e imagen como para promover cambios sociales positivos debido a que son modelos de vida e importantes influenciadores.

De hecho, el deporte congrega a personas de diferentes orígenes incluso de diferentes culturas, contribuyendo a fomentar el desarrollo de sociedades multiculturales y compasivas (Janssens \& Verweel, P., 2014), lo que remite a situaciones en las que algunos miembros de organizaciones deportivas lograron mejores indicadores en temas relacionados con el capital social como lo son la percepción de confianza en el gobierno, mayor tolerancia y mejores relaciones con sus vecinos (Brown et al., 2014).

De igual manera, Martínez et al. (2021) encontraron en usuarios de la ciclovía la mayor tasa de satisfacción ciudadana frente a un programa gubernamental, además de una importante estrategia de política pública para incentivar hábitos saludables, cohesionar el tejido social e incrementar la percepción de confianza en el gobierno.

Para Pye et al. (2015) cuando no se planifican los beneficios sociales de una Ciudad Deportiva esto se traduce en el decrecimiento del aporte social a través del deporte además de la falta de articulación entre la infraestructura deportiva y las necesidades de la comunidad.

\section{Infraestructura y equipamiento deportivo}

Las ciudades deportivas deben planificar la infraestructura deportiva y el equipamiento deportivo a largo plazo, esto es debido a que son trascendentales para el desarrollo de una Ciudad Deportiva porque se convierten en los instrumentos que permiten diferentes prácticas deportivas. Por esto, para los componentes clave de una ciudad habitable o «viva»: la seguridad de la ciudad, la vivacidad, la sostenibilidad y salud de la ciudad; la infraestructura deportiva tiene un rol fundamental en cada uno de ellos (Gehl, 2006, 2010, 2011).

Acerca de la vivacidad, Gehl (2010) afirma que donde abundan las instalaciones deportivas, el horizonte de 
actividades opcionales para la comunidad se amplía y el número potencial de actividades sociales se incrementa por lo que debe existir una conexión entre planificación urbana y justicia social. Coaffee \& Shaw (2005) están de acuerdo y expresan que el deporte ha incrementado su participación en la planificación estratégica de una ciudad global moderna. Al respecto, Smith (2010) sostiene que el desarrollo de las ciudades deportivas, cualquiera que sea su forma de expresión, debe planificarse y gestionarse especialmente si se buscan impactos sociales a largo plazo o duraderos. Estratégicamente, esto incluye una planificación urbana eficaz (Gehl, 2010), la integración de programas sociales (Coalter, 2005) y la formación de asociaciones eficaces (Coalter, 2005; Misener \& Mason 2010).

De la dimensión social del espacio público hablan Borja \& Muixí (2001, p. 47) cuando expresan: «Es un lugar de relación y de identificación, de contacto entre las personas, de animación urbana, a veces de expresión comunitaria». Además, pueden ser un factor coadyuvante en la equidad de género debido a que cuando las mujeres hacen uso de los espacios públicos, a menudo lo hacen sin desvincularse de su rol familiar y es en el deporte donde «Encuentran una manera de borrar la tradicional división entre ámbito público y familiar» (Vilanova \& Soler, 2008, p.30). Para Latham \& Layton (2020) las buenas ciudades requieren una extensa y robusta infraestructura social con acceso universal para facilitar la conexión y permitir que las personas y las comunidades prosperen.

Es así porque las infraestructuras deportivas comunitarias de las que por lo general el gobierno es el único proveedor, permiten el acceso a la práctica deportiva cotidiana y como importantes artilugios de la infraestructura socio-cultural, incrementan el desarrollo social debido a que cimentan entornos que facilitan y forjan la construcción de identidad en la comunidad y promueven el entendimiento mutuo; debido a esto, las ciencias sociales tienen la necesidad de investigar para realizar un registro prospectivo que les permita volver a imaginar, volver a describir y defender la creación de mejores futuros urbanos (Simone \& Pieterse (2017).

\section{Capital Humano y Gestión del Conocimiento.}

En la Ciudad Deportiva es un factor clave que debe entenderse como la capacidad del sector deporte, recreación y actividad física (DRAF) para generar nuevos saber, divulgarlo entre los stakeholders o materializarlo en productos y servicios. A través de este proceso la ciudad innova (Nonaka, 2000) y facilita la mirada estratégica de la gobernanza, la administración y la evaluación del modelo de ciudad deportiva implementado. En este punto, el capital humano se convierte en una oportunidad/fortaleza o brecha que se debe gestionar.

Respecto al capital humano, el flujo constante de información en la Ciudad Deportiva forja un mercado laboral en constante movimiento por la creación permanente de nuevas demandas que requieren nuevas destrezas, competencias y habilidades que tienen que ser suplidas por trabajadores cada vez mejor formados. Para Mayer \& Cortright (2012,p.268), parte interesante de este capital son los «conocedores apasionados», empleados o empresarios que utilizan los productos y servicios del deporte y ofrecen importante información acerca de estos. Las brechas se dan cuando el sector productivo demanda ciertas competencias en el mercado laboral que son difíciles de suplir, cuando la cantidad de trabajadores no es suficiente o cuando se afecta el bienestar del trabajador, todo esto en detrimento de la productividad y la competitividad. Por esto se requieren procesos de articulación entre la academia y el sector empresarial del deporte, además de la regulación de políticas públicas y privadas que incrementen el bienestar del capital humano del sector productivo del deporte.

\section{Deporte y Desarrollo Económico}

Se reconoce la relevancia de la contribución del sector deporte para la economía de la Ciudad Deportiva, no obstante, en muchos países se desconoce su comportamiento y contribución al producto interno bruto (PIB); debido a esto el sector carece de un marco económico conceptual unificado, un sistema de información periódico y una estadística confiable que permita conocer los resultados económicos del sector y las posibles implicaciones que tienen las políticas económicas en el mismo (Pedrosa, Salvador, 2003). Por lo tanto, un paso sustancial que han desarrollado algunos países como Reino Unido, Alemania y España ha sido realizar mediciones periódicas acerca de la contribución del sector, a través de iniciativas tendientes a la construcción de cuentas satélites, agrupando flujos económicos del sector dando respuesta a las preguntas: «¿Quién produce?, ¿Quién financia? y ¿Quién se beneficia?» (Ruiz, et al., 2010,p.149).

De igual manera, estrategias en torno al reconocimiento de la productividad y la competitividad y formas novedosas para dinamizar el sector tales como: iniciativas clusters, hub de emprendimiento, revitalización 
y nuevos usos de la infraestructura deportiva, aceleradoras, y el diseño de políticas relacionadas con incentivar o cerrar brechas en aspectos que afectan indicadores económicos relevantes para dicho sector.

Respecto a los clusters Shilbury (2000) afirma que estos facilitan la especificidad en la industria del deporte, maximizar las contribuciones financieras de organizaciones que dependen del éxito continuo de un deporte en el mercado, cambiar el sistema de prestación de servicios deportivos, visibilizar el amplio rol que tienen las organizaciones deportivas nacionales y estatales, definir los vínculos entre proveedores y compradores y aprovechar el apoyo financiero dentro del grupo cluster para complementar el apoyo gubernamental que si bien existe es limitado. Todo esto hace necesario la realización de un mapeo constante de las interacciones entre industrias y organizaciones para comprender mejor las redes de clústeres y la competencia.

Las dificultades se dan debido a la complejidad de la industria del deporte la cual está dada por ser el sector DRAF uno de los denominados sectores transversales donde se agrupan actividades diferentes que tienen un objetivo único común: el consumidor. En este sentido, Mullin et al. (1995) describen el producto deporte desde la amplitud de los beneficios ofrecidos tanto a usuarios como espectadores y señalan como los componentes primarios del producto son: forma de juego por sí misma, acontecimiento y sus estrellas, boletería, organización, instalaciones, material, vestuario y modas, y otros servicios como el personal, los procesos y la imagen.

El deporte tiene cuatro dominios a saber: los espectadores, la participación en los deportes, los bienes del deporte (equipamiento deportivo, ropa deportiva, calzado deportivo y muchos otros productos relacionados con el deporte como souvenirs, clases, bebidas, alimentos, formación, seguros, entre otros) y las categorías de productos no deportivos o aquellos que utilizan el deporte como plataforma o medio para comercializar sus productos (Fullerton \& Russell, 2008).

Acerca de la venta de servicios deportivos y bienes relacionados por parte de entidades (organizaciones, personas autónomas y sociedades) que ofrecen deporte a participantes y espectadores, dicho sector a nivel global alcanzó cifras cercanas a US\$ 388.3 billones en 2020 (72\% deporte participativo), con una tasa de crecimiento anual compuesta del 3.4\% desde 2015. El mercado disminuyó de US\$ 458.8 billones en 2019 a \$ 388.3 billones en 2020 (-15.4\%) debido al distanciamiento social y la desaceleración económica ocasionada por la enfermedad de COVID-19 y las acciones para contenerla. Se espera que el mercado alcance los \$ 599.9 mil millones para el 2025 y \$ 826 billones para el 2030 (The Business, 2019). Estas cifras a pesar de ser parciales, por no abarcar todos los dominios del deporte, muestran el alcance económico del sector y sus posibilidades de crecimiento.

Respecto a la pandemia como experiencia sin precedentes, esta fue apropiada para reevaluar los modelos de negocio y construir un nuevo futuro en el que se desarrollen fuertes conexiones con los clientes, analítica de datos, big data, adquirir capacidades digitales, consecución de ingresos innovadores (Deloitte, 2020). Tecnología de última generación como Internet of Technologies (IoT) permitirán mejorar la experiencia de los espectadores en los estadios y arenas y fortalecer a los equipos, siendo los E-deportes, apuestas en línea, el uso de realidad virtual nuevas tendencias que dinamizarán el sector (The Business, 2019)

Con relación a la competencia que se da entre las marcas deportivas nacionales y las marcas internacionales, Zhang, Zhang \& Guo (2020) proponen aumentar la inversión en investigación y desarrollo (I + D) mediante la creación de clústeres industriales donde se reúnan empresas pequeñas y medianas con empresas más grandes para desarrollar productos de alta gama, vinculando a las universidades o instituciones de investigación, para mejorar la competitividad del mercado de los productos deportivos desde los aspectos medioambientales protección, economía, durabilidad, comodidad, belleza y multifunción; mejorando con ello la autoconfianza nacional en sus marcas deportivas.

Capítulo aparte dentro de esta industria, merece el turismo deportivo por la importancia que tiene para el desarrollo y posicionamiento de la ciudad que corresponda, cuyas actividades se pueden clasificar como eventos deportivos turísticos, turismo deportivo de nostalgia, turismo deportivo activo (Gibson, 2002). Es una de las tendencias que mayor crecimiento le ha representado al turismo mundial, aunque Herbold et al. (2020), refieren que las comunidades, especialmente las de países en desarrollo, aún no comprenden la importancia y el potencial del deporte local, como recurso y ventaja competitiva en el desarrollo de un destino turístico. Así, para Cabanilla et al. (2021), los grandes eventos deportivos tienen la capacidad de atraer turistas e impactan de manera inmediata la cadena de valor del turismo, Leal et al. (2021) recomienda mejorar la coordinación entre stakeholders: autoridades del deporte y el turismo, agentes del turismo y el deporte, políticas públicas 
de ambos sectores.

\section{Grandes Eventos Deportivos}

Los eventos deportivos a gran escala, especialmente los megaeventos, fueron a partir de la década de 1980 para las ciudades anfitrionas, novedosos impulsores del crecimiento de la economía, a medida que los panoramas urbanos se transformaron de centros industriales de producción a centros de ocio para el consumo (Giampiccoli et al., 2015) tal es el caso de Cardiff y Manchester (Smith 2010).

Un rasgo común de las ciudades deportivas es su origen, infaliblemente conectado con grandes acontecimientos deportivos ya sea como preparación para la candidatura a un gran evento como Manchester, o como producto de un mega evento deportivo, tal es el caso de Barcelona. De ahí radica que el presente modelo de Ciudad Deportiva proponga la realización de eventos deportivos como factor primordial; para Misener \& Mason (2008) conlleva dos implicaciones: utilizar el evento para confirmar la imagen de crecimiento de la urbe o para posicionar la marca de ciudad dentro del competitivo entorno global.

De otro lado, los eventos le dan a la ciudad un ambiente de emoción y estímulo, una sensación de festivalización; término utilizado para describir el «sentirse bien», además de orgullo cívico debido al sentido de entusiasmo creado por el suceso, proporcionando entornos en los que la ciudad puede integrar su función deportiva con sus festivales culturales (Pye et al., 2015, Richard \& Palmer, 2010). Para Rio de Janeiro 2016 implicó el aumento de la reputación y exposición internacional (Barogui et al., 2021).

Es así como un gran evento debería estar incluido en los planes generales de desarrollo de la Ciudad Deportiva y su viabilidad deber ser parte de la planificación, lo mismo que la evaluación parte de la gestión post-evento. Además de ser objeto de una profunda implicación de todos los actores sociales y económicos con interés en el evento (Vico, 2018).

\section{Cuarto Usuario/beneficiario}

Como se sabe, la planificación de una Ciudad Deportiva debe equilibrar necesidades sociales, económicas y ambientales comunitarias, mientras maximiza el potencial económico de la industria del deporte mundial, siempre en constante crecimiento (Pye et al., 2015). Surge en la Ciudad Deportiva la necesidad de identificar los tipos de clientes a los cuales la Ciudad Deportiva requiere satisfacer los cuales se pueden dividir de mu- chas maneras como la que se propone a continuación.

- Atletas/deportistas/staff,

- Espectadores/seguidores/audiencia,

- Patrocinadores/inversores

- Clientes corporativos.

Esta nueva manera de reconocer en los usuarios un cliente al que se quiere satisfacer le dan un enfoque de servicio y calidad del servicio que crea una nueva manera de abordar los diferentes grupos de interés que conforman la Ciudad Deportiva.

\section{Quinto Impacto}

El impacto visto como las consecuencias de impulsar un modelo de Ciudad Deportiva, se encuentran relacionado con todos y cada uno de los componentes del modelo, su evaluación deberá informar los diferentes cambios observados consecuencia de la aplicación del modelo, por esto este modelo propone la creación de indicadores relacionados con los Objetivos de Desarrollo Sostenible, especialmente los relacionados con las ODS 3, 4, 5, 10, 11 y 16 .

\section{Objetivos de Desarrollo Sostenible (ODS)}

El efecto se relaciona con el deporte como un medio que contribuye al cumplimiento de los Objetivos de Desarrollo Sostenible. Desde la promulgación de la Agenda 2030 para el Desarrollo Sostenible por Naciones Unidades (ONU) en el 2015 los cuales pretenden impulsar el cambio de la sociedad, se hace indispensable que los diferentes actores del deporte establezcan indicadores relacionados con el cumplimiento de los ODS, teniendo en cuenta el reconocimiento que se ha hecho del deporte como importante facilitador del desarrollo sostenible y la paz.

Por esto se hace necesario articular las ODS en los planes de desarrollo, en las políticas públicas, planes estratégicos y como indicadores de los planes de acción, realizando trabajo mancomunado con los actores del Deporsistema (sociedad civil, empresas privadas, academia, entidades públicas).

\section{Conclusiones}

Este documento es una más de las evidencias de la importante transversalidad del deporte, para lograr bajo la gestión institucional, el bienestar biopsicosocial y económico además del desarrollo comunitario. Pretende ser una herramienta didáctica, organizacional, incluso social, proyectada para fundamentar la gestión administrativa basada en el deporte, unificando, además, el de- 
ber ser de las instituciones delegatarias del Estado en las cuales se desarrollan procesos confluyentes en la optimización de la calidad de vida social con la fuerza del deporte para realizar acciones transversales.

Se concluye que el deporte tiene méritos suficientes para convertirse en el centro y el artífice de la especialización inteligente de toda una ciudad o un territorio previamente especificado de ella. Es por esto que se han encontrado múltiples organizaciones que registran a las ciudades bajo denominaciones específicas, etiquetándolas como «ciudades deportivas» y otros nombres relacionados, sin embargo, no se encontró unanimidad al respecto ni una definición de lo que puede ser una Ciudad Deportiva. Lo que sí se puede aseverar es como la realización de grandes eventos y megaeventos deportivos y sobre todo las facilidades que se dan en torno a la organización del evento se constituyen en una de las mejores prácticas para lograr estas etiquetas.

La información recopilada y analizada permitió generar un instrumento proyectivo, facilitador del aseguramiento de estrategias que guiarán a los stakeholders del deporte, en el diseño de un modelo propio adaptado a su contexto, necesidades y deseos de la población, además de los de la gobernanza.

\section{Aplicaciones prácticas}

Para aquellas ciudades que en honor a su pujanza luchan día a día para crecer de manera sustentable reconociendo las bondades de la práctica deportiva, la aplicación de este modelo propuesto les permitirá planificar la manera de interactuar con cada uno de los componentes del modelo para perfilar las diferentes estrategias que han hecho, hacen y harán única y especial a una Ciudad Deportiva.

\section{Futuras Líneas de Investigación}

A futuro, profundizar en el conocimiento de las políticas públicas de las denominadas «ciudades deportivas» permitirá reconocer cuales son las estrategias fundamentales llevadas a cabo por dichas ciudades para posicionarse como ciudades deportivas. De igual manera la realización de estudios en las ciudades deportivas relacionados con el logro de los Objetivos de Desarrollo Sostenible a través de la herramienta socio-económica denominada deporte permitirá visibilizar el sector DRAF, como lo que es, un medio para el incremento y mejoramiento del bienestar de la sociedad.

En este objetivo se hace necesario la realización de estudios de carácter científico: trabajos de investigaciones básicas y/o aplicadas, incluso divulgaciones como lo pueden ser el intercambio de propuestas y experiencias investigadas.

\section{Referencias}

ACES Europe (2021). Candidature. http://aceseurope.eu/ candidature-2/.

América Retail (2021,19 de enero). Retail Deportivo: el deporte, rey del retail en EEUU.América Retail.https:/ / www.americaretail.com/retail-deportivo/retail-deportivo-el-deporterey-del-retail-en-eeuu/\#.

ATKearney (2014).Winning in the Business Sport. Collington, $\mathrm{H}$. \& Sultan, N. https://www.kearney.com/communicationsmedia-technology/article?/a/winning-in-the-business-ofsports.

Cabanilla, E., Lastra-Bravo, X., Pazmiño, J.P.; Burbano, M.(2021). Análisis del perfil demográfico y consumo turístico en eventos deportivos en la ciudad de Quito. Caso de estudio: Roger Federer. Retos, 40, 2. https://recyt.fecyt.es/index.php/retos/article/view/82749/62138.

Barogui, Riveiro \& Lourenco (2021). Experts' Opinions about the Sustainability Impact Intensity of the Olympics in Rio de Janeiro. Journal of Urban Planning and Development, 147:1,1. https:/ / ascelibrary.org/doi/abs/10.1061/ \%28ASCE\%29UP.1943-5444.0000618.

Biernat, E., NaBêcz, H., Skrok, A., \& Majcherek, D. (2020). Do Sports Clubs Contribute to the Accumulation of Regional Social Capital?. International journal of environmental research and public health, 17(14), 5257. https://doi.org/10.3390/ ijerph17145257.

Brown, K.M , Hoye R. \& Nicholson M. (2014). Generating trust? Sport and community participation. Journal of Sociology, 50(4):437-457. doi:10.1177/1440783312467091.

Borja,J.\& Muixí,Z. (2001). Espai públic: ciutati ciutadania. Barcelona: Diputació de Barcelona.

Burson Cohn \&Wolfe (2021).2021 ranking of sports cities. https:/ /bcw-global.com/documents / 99/Report__BCW_Ranking_of_Sports_Cities_2021_FINAL.pdf.

Camy, J.; Comité Olímpico Internacional; Solidaridad Olímpica (2008). Gestión de las Organizaciones Deportivas Olímpicas. Human Kinetics; Solidaridad Olímpica.

Castillo-Retamal, F., Matus-Castillo, C., Vargas-Contreras, C., Canan, F., Starepravo, F., \& Bássoli de Oliveira,A. (2020). Participación ciudadana en la elaboración de las políticas públicas de actividad física y deporte: el caso de Chile. Retos, 38,482489. https://doi.org/10.47197/retos.v38i38.76340

Centro de Estudios Olímpicos (2015). Juegos de la XVII Olimpiada, Roma 1960. https: / / estudiosolimpicos.es/index.php/ olimpismo/historia-olimpica/olimpiadas-modernas/item/ 44-juegos-de-la-xvii-olimpiada-roma-1960.

Coaffee, J., \& Shaw,T. (2005). Viewpoint:The Liveability Agenda: New Regionalism, Liveability and the Untapped Potential of 
Sportand Recreation. TheTown Planning Review, 76(2), I-V.http:/ /www.jstor.org/stable/40112637.

Coalter, F. 2005. The Social Benefits of Sport: An Overview to Inform the Community Planning Process. Sportscotland Research Report 98. Edinburgh: Sportscotland.

Coalter, F. 2007a. «Sports Clubs, Social Capital and Social Regeneration: 'Ill-Defined Interventions with Hard to Follow Outcomes'?». Sport in Society 10 (4): 537-559. doi:10.1080/ 17430430701388723.

Coalter, F. 2007b. A Wider Social Role for Sport:Who's Keeping the Score? London: Routledge.

Coalter, F. 2010. «The Politics of Sport-for-Development:Limited Focus Programmes and Broad Gauge Problems?» International Review for the Sociology of Sport 45 (3): 295 314. doi:10. 1177/1012690210366791.

Chappelet, J-L., Mrkonjic,M. (2013). Action for Good Governance in International Sports Organisations (AGGIS). Final report. Copenhagen: Play the Game/Instituto Danés de Estudios Deportivos. https: / / playthegame.org/theme-pages/thesports-governance-observer/about-the-project/ . https: / / www.wada-ama.org/en/what-we-do.

Deloitte (2020). 2021 outlook for the US sports industry. Interview with Pete Giorgio. https://www2.deloitte.com/content/ dam/Deloitte/us / Documents / technology-mediatelecommunications/us-tmt-sports-outlook-2021.pdf.

Donnelly, P. (2010). Sport and social theory. Sport and Society: A student introduction, 11-32.

dos Anjos, V. Á. A., Tavares, G. H., Ferreira, J. S., \& da Silva, J.V. P. (2021). Políticas públicas de promoção de saúde e orientação profissional em AAL em Campo Grande/MS, Brasil (Public health promotion policies and professional guidance in the Outdoor Gyms in Campo Grande/MS, Brazil). Retos, 41, 112 122.https://recyt.fecyt.es/index.php/retos/article/view/ 83012.

Felfe C., Lechner M. \& Steinmayr A. (2016).Sports and Child Development. PLoS One, 4;11(5):e0151729. doi: 10.1371/ journal.pone.0151729. PMID: 27144474; PMCID: PMC4856309.

Gehl, J. 2006. New City Spaces. Copenhagen: The Danish Architectural Press.

Gehl, J. 2010. Cities for People. Covelo, CA: Island Press.

Gehl, J. 2011. Life Between Buildings. Washington, DC: Island Press.

Geeraert,A. (2015). Observador de la Gobernanza del Deporte. La crisis de legitimidad en la gobernanza deportiva internacional. Play the Game/Instituto Danés de Estudios Deportivos/ Universidad de Leuven.

Gerkea, A., Babiak, K.,Dickson, G., Desbordes, M. (2018). Developmental processes and motivations for linkages in cross-sectoral sport clusters. Sport Management Review 21, 133 146.

Giampiccoli,A., Lee, S. S., \& Nauright, J. (2015). Destination South Africa: Comparing global sports mega-events and recurring localised sports events in South Africa for tourism and economic development. Current Issues in Tourism, 18(3), 229248.

Gibson, H. (2002). SportTourism:A Critical Analysis of Research. Sport Management Review, 1(1), 45-76. doi:https://doi.org/ 10.1016/S1441-3523(98)70099-3.

Sportcal (2021). Global sports (GSI), cities index. GSI Cities Index | GSI | Sportcal.

Gold, J.R. \& Ward, S.V. (1994): Place promotion. The use of publicity and marketing to sell towns and regions, Chichester, John Wiley \& Sons.

Herbold, V., Thees, H. , Philipp, J. (2020). The Host Community and Its Role in Sports Tourism - Exploring an Emerging Research Field. Sustainability, 12, 24, 1-26. https://doi.org/ $10.3390 /$ su122410488.

Janssens, J., Verweel, P. (2014). The significance of sports clubs within multicultural society. On the accumulation of social capital by migrants in culturally «mixed» and «separate» sports clubs. Eur.J. Sport Soc., 11, 35-58.

Fullerton, S., \& Merz, G. R. (2008). The four domains of sports marketing: A conceptual framework. Sport Marketing Quarterly, 17(2), 90-108. https://commons.emich.edu/ mktg_facsch/26/.

Gratton, C., Shibli, S., \& Coleman, R. (2005). Sport and Economic Regeneration in Cities. Urban Studies;42(5-6):985-999. doi:10.1080/00420980500107045.

Iria, P. (2021, enero, 19). El deporte, rey del retail en EEUU: sólo comida y hogar le superan en 2020. Palco23.El deporte, rey del retail en EEUU: sólo comida y hogar le superan en 2020 | Palco23.

Kearney (2020). Global Cities Report. 2020. Global Cities Index: New priorities for a new world. https: / / www.kearney.com/globalcities/2020.

Kharytonov,Y.O., Kharytonov, E., Kharytonova, Î.I., Kostruba,A.V., Tkalych,M.Î., \&Tolmachevska,Y.O. (2020).To the Peculiarities of Legal and Non-Legal Regulation of Social Relations in the Field of Sport. Retos, 41, 131-137. https://doi.org/ 10.47197/retos.v0i41.84178.

Landabasco, M. (2015). La especialización inteligente es un planteamiento estratégico de la Unión Europea para dar un nuevo rumbo a su política de cohesión territorial. Organización de las Naciones Unidas para la Alimentación y la Agricultura (FAO). http: / /www.fao.org/in-action/territorios-inteligentes/articulos/ opiniones-territoriales/detalle/es/c/1330679/.

Latham, A., \& Layton, J. (2020). Kinaesthetic cities: Studying the worlds of amateur sports and fitness in contemporary urban environments. Progress in Human Geography, 44(5), 852-876.

Latiesa, M., \& Paniza, J.L. (2006). Turistas deportivos. Una perspectiva de análisis. Revista Internacional de Sociología (RIS), 133 149. https://doi.org/10.3989/ris.2006.i44.31.

Leal, M., Travé, R., Medina, F.X.,Abadia, S., \& Sánchez, S. (2021). Turismo deportivo: Una oportunidad para los territorios locales en un contexto global. Un estudio Delphi (Sport tourism: an opportunity for local regions in a global context. A Delphi study). Retos, 42, 77-88. https://doi.org/10.47197/ 
retos.v42i0.86696

Martínez, L., Trofimoff, V., \& Zafra, M.I. (10 de junio de 2021). Mejorando la percepción gubernamental. Ciclovida, una herramienta pública para incentivar hábitos saludables y cohesionar el tejido social [Youtube]. Webinar Académico CALI CIUDAD DEPORTIVA - YouTube.

Mayer,H., \& Cortright, J. (2012).The role of culture, consumption and community in cluster development: the case of Portland's athletic and outdoor industry, Oregon (USA). Geographica Helvetica, 66(4), 261-270.

Misener, L., \& Mason, D. (2010). Towards a Community Centred Approach to Corporate Community Involvement in the Sporting EventsAgenda. Journal of Management and Organization, 16 (4), 495- 514. doi:10.5172/jmo.2010.16.4.495.

Misener, L. \& Mason, D. (2008). Urban Regimes and the Sporting Events Agenda: A CrossNational Comparison of Civic Development Strategies. Journal of Sport Management 22 (5), 603-627.

Misener, L. \& Mason. D. (2010). Towards a Community Centred Approach to Corporate Community Involvement in the Sporting EventsAgenda. Journal of Management and Organization, 16 (4), 495- 514. doi:10.5172/jmo.2010.16.4.495.

.Press of Southern Denmark.

Mullin, B., Hardy, S., \& Sutton, W. (1995). Marketing Deportivo. Paidotribo.

Muñoz,F.(1997). Historic evolution and urban planning typology of OlympicVillages. Centre d'Estudis Olímpics, 27-51.https:/ /core.ac.uk/download/pdf/13282887.pdf.

Nauright, J., \& Zipp, Z. (2018). The complex world of global sport. Sport in Society, 21(8), 1113-1119.https://doi.org/ 10.1080/17430437.2018.1469846.

Nonaka, I. (2000). Gestión del Conocimiento. La Empresa Creadora de Conocimiento. Harvard Business Review. Deusto.

Pye, P., Toohey, K., \& Cuskelly, G. (2015). The social benefits in sport city planning: a conceptual framework, Sport in Society, 18(10), 1199-1221, DOI: 10.1080/ 17430437.2015 .1024235$.

Shilbury, D. (2000). Considering future sport delivery systems. Sport Management Review, 3(2), 199-221. Considering Future Sport Delivery Systems - ScienceDirect

Skrok, L., Majcherek, D., NaBêcz, H., \& Biernat, E. (2019). Impact of sports activity on Polish adults: Self-reported health, social capital \& attitudes. PLoS ONE, 14(12), e0226812. https:/ / doi.org/10.1371/journal.pone.0226812.

Sportbusiness (2018). Ultimate Sports City Reports. Ultimate Sports City 2018 | SportBusiness.

Simone, A., \& Pieterse, E. (2018). New urban world: Inhabiting dissonant times. John Wiley \& Sons.

Smith,A.(2005a). Reimaging the city:The value of sport initiatives. Annals of tourism research, 32(1), 217-236. https://doi.org/ 10.1016/j.annals.2004.07.007.

Smith, A. (2005b). Conceptualizing city image change: The reimaging of Barcelona, Tourism Geographies, 7(4),398- 423. DOI: $10.1080 / 14616680500291188$.

Smith,A. (2010). The Development of «Sports-City» Zones and
Their Potential Value as Tourism Resources for Urban Areas, European Planning Studies, 18(3), 385410. DOI: 10.1080/09654310903497702.

Strauss, A., \& Corbin, J. (2003). Bases de la investigación cualitativa técnicas y procedimientos para desarrollar la teoría fundamentada. Universidad de Antioquia. Bases_de_la_investigacion_cualitativa_Strauss_y_Corbinwith-cover-page-v2.pdf (d1wqtxts1xzle7.cloudfront.net).

The Business (2019). Sports Market By Type (Participatory Sports, Spectator Sports, Sports Team \& Clubs, Racing \& Individual Sports And Spectator Sports), By Competitive Landscape And By Geography Global Forecast To 2022. https:// www.thebusinessresearchcompany.com/report/sportsmarket.

UNESCO \& MINEP VI (2017). Plan de Acción de Kazán. Sexta Conferencia Internacional de Ministros y Altos Funcionarios de Deporte, MINEPVI. https: / / es.unesco.org/mineps6/kazanaction-plan.

UNESCO (2020). UNESCO for Sustainable Cities.https: / / en.unesco.org/unesco-for-sustainable-cities.

Vico, R.P.(2018).Turismo de eventos, mega-eventos y gobernanza territorial. Revista Geográfica de Valparaiso, 55,1-20. http:/ / www.revistageografica.cl/index.php/revgeo/article/view/ 22.

Vilanova, A. \& Soler, S. (2008). Las mujeres, el deporte y los espacios públicos: ausencias y protagonismos. Apunts Educación Física y Deportes, 91, 29-34. Las mujeres, el deporte y los espacios públicos: ausencias y protagonismos - INEFC (revista-apunts.com).

Whitson, D. (2004). Bringing theWorld to Canada:The Periphery of the Centre'. ThirdWorld Quarterly, 25(7), 1215-1232. http:/ /www.jstor.org/stable/3993806.

UNWTO (2016). Unwto International Conference on Tourism and Sports. Da Nnang declaration on promoting tourism and sports for sustainable development. UNWTO Internacional Conference on tourism and sports. Da Nang (Vietnam). UNWTO International Conference on Tourism and Sports | UNWTO.

Zhang, HL., Zhang, HJ., \& Guo, XT. (2020). Research on the future development prospects of sports products industry under the mode of e-commerce and internet of things. InfSyst E-Bus Manage, 18, 511-525. https://doi.org/10.1007/ s10257-018-0382-2.

Zhelyazkov, T. (2006). Teoría y Metodología del Entrenamiento Deportivo. Escuela Nacional del Deporte. 